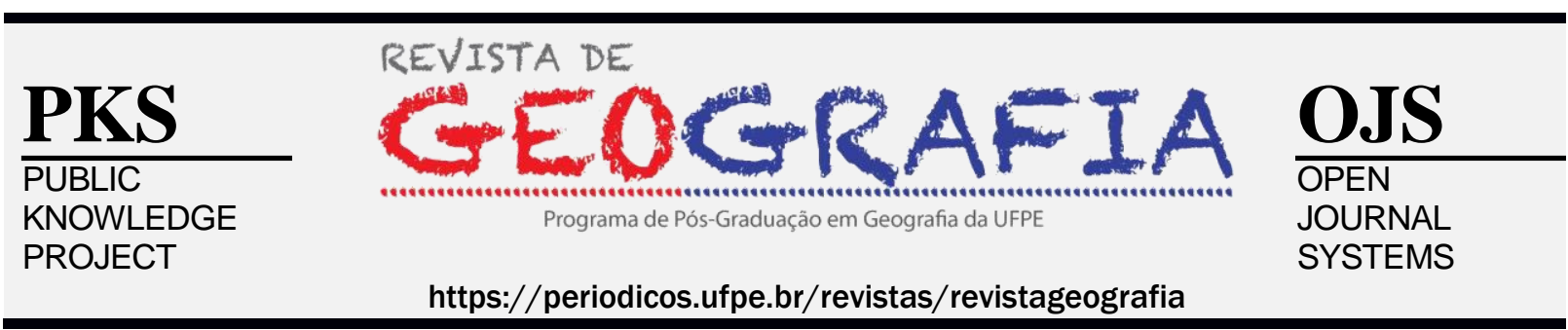

\title{
ANÁLISE ESTATÍSTICA PARA DETECÇÃO DE TENDÊNCIAS EM SÉRIES TEMPORAIS DE TEMPERATURA E PRECIPITAÇÃO NO RECIFE-PE
}

\author{
Marcos Antonio Barbosa da Silva Junior ${ }^{1}$, Gastão Cerquinha da Fonseca Neto², Jaime \\ Joaquim da Silva Pereira Cabral ${ }^{3}$
}

\author{
${ }^{1}$ Doutorando em Engenharia Civil pela UFPE. E-mail: marcos15barbosa@ hotmail.com \\ ${ }^{2}$ Doutorando em Engenharia Civil pela UFPE. E-mail: gastaocerquinha@gmail.com \\ ${ }^{3}$ Professorna UFPE e UPE. Email: jcabral@ufpe.br
}

Artigo recebido em 30/12/2018 e aceito em 22/11/2019

\begin{abstract}
RESUMO
O presente trabalho buscou identificar tendências nas séries temporais de temperatura e precipitação na cidade do Recife-PE. Para isso, foram utilizados os dados diários, mensais e anuais da estação climatológica Recife (Curado), operada pelo INMET, entre 1961 e 2017. As tendências foram detectadas por meio de regressão linear simples e testes de Mann-Kendall (com níveis de significância de 5\% e 10\%) e Curvatura de Sen, usando o software estatístico XLSTAT 2018. Os resultados mostraram tendência positiva para as séries temporais de temperaturas mínima, média e máxima (mensal e anual). Nas séries de precipitações máximas e totais (mensal e anual), a regressão linear simples não apontou tendência nos dados, diante dos baixos coeficientes de determinação. Em contrapartida, os testes de Mann-Kendall e Curvatura de Sen mostraram que, apenas para os meses de março e setembro, foi detectada tendência negativa e estatisticamente significante nas séries temporais dos totais precipitados. Já a ocorrência de eventos com precipitações extremas $(\geq 100 \mathrm{~mm} / 24 \mathrm{~h})$ apresentou tendência positiva e levemente significante.
\end{abstract}

Palavras-Chave: Clima; Regressão linear simples; Mann-Kendall; Curvatura de Sen.

\section{STATISTICAL ANALYSIS FOR DETECTION OF TRENDS IN TEMPERATURE AND PRECIPITATION TIME SERIES IN RECIFE-PE}

\begin{abstract}
This paper sought to identify trends in the time series of temperature and precipitation in the city of Recife-PE. For this, the daily, monthly and annual data of the Recife (Curado) climatological station operated by INMET between 1961 and 2017 were used. Trends were detected through simple linear regression and Mann-Kendall tests (with of 5\% and 10\% significance) and Sen Curvature, using XLSTAT 2018 statistical software. The results showed a positive trend for the minimum, average and maximum (monthly and annual) time series. In the series of maximum and total precipitations (monthly and annual), simple linear regression did not indicate trend in the data, due to the low coefficients of determination. In contrast, the Mann-Kendall and Curvatura de Sen tests showed that for the months of March and September only a negative and statistically significant trend was detected in the time series of precipitated totals. On the other hand, the occurrence of events with extreme precipitations $(\geq$ $100 \mathrm{~mm} / 24 \mathrm{~h}$ ) presented a positive and slightly significant trend.
\end{abstract}

Keywords: Climate; Simple linear regression; Mann-Kendall; Sen's curvature. 


\section{INTRODUÇÃO}

Associado ao processo de urbanização acelerado e não planejado, observado nas grandes metrópoles brasileiras, outro fator relevante e de grande preocupação para a sociedade são os efeitos provocados pelas alterações climáticas globais. De acordo com o quinto relatório de avaliação do Painel Intergovernamental de Mudanças Climáticas (IPCC, 2014), num cenário mundial, os riscos relacionados aos eventos climáticos extremos, tais como precipitação intensa e inundações costeiras (elevação do nível do mar), já se mostram de moderado a alto. Esses eventos, que já se mostram trágicos e desastrosos nas grandes capitais devido ao uso inadequado do solo e de técnicas pouco eficientes de drenagem, podem ser agravados em um cenário futuro de ciclos hidrológicos alterados (MIGUEZ et al., 2011; SILVA JUNIOR; SILVA, 2016).

Numa escala local ou regional, as mudanças climáticas são detectadas por meio de análise de tendências nas séries históricas das variáveis meteorológicas, como temperatura e precipitação. Para isso, são necessários registros climáticos de qualidade e a longo prazo (SOUZA; AZEVEDO, 2012). Salviano, Groppo e Pellegrino (2016) ressaltam que as mudanças identificadas nas séries temporais de uma variável climatológica, podem estar associadas à diversos fatores de origem, como: antrópico, por meio dos desmatamentos e da urbanização desenfreada; ou natural, através da intensificação da atividade solar e anomalia climática como o El Niño e La Niña.

Uma das formas mais simplificadas de se obter tais tendências climáticas, é fazendo uso de métodos estatísticos, com a aplicação de testes paramétricos e não paramétricos. Vários pesquisadores brasileiros têm empregado tal método para identificar tendências em séries temporais de temperatura e precipitação, além de avaliar a sua significância estatística (MESQUITA et al., 2017; NUNES; PINTO; BAPTISTA, 2018; PINHEIRO; GRACIANO; SEVERO, 2013; ROCHA et al., 2017; SALVIANO; GROPPO; PELLEGRINO, 2016; WANDERLEY; JUSTINO; SEDIYAMA, 2016).

Além dos métodos estatísticos, o uso de softwares para identificação de tendências climáticas também é bastante comum e, dentre os quais, se destaca o RClimdex. Algumas pesquisas no intuito de detectar tendências em séries de variáveis climatológicas (temperatura e precipitação) em localidades do Estado de Pernambuco (Recife e outras cidades) já empregaram o citado software, o qual é utilizado, basicamente, para o processamento de dados 
e obtenção dos índices climáticos (SILVA; MONTENEGRO; SOUZA, 2017; SOUZA; AZEVEDO, 2009; SOUZA; AZEVEDO, 2012).

Com enfoque no emprego de métodos estatísticos para a análise de tendências em séries climatológicas em Pernambuco, Medeiros, Holanda e Silva (2018) identificaram as tendências lineares e a variabilidade do coeficiente de regressão nas séries mensais de precipitação no município de São Bento do Una (Agreste Pernambucano), no período de 1920 a 2016. Para o Recife, Wanderley et al. (2018) investigou as frequências, as tendências e o tempo de recorrência dos eventos pluviométricos extremos ocorridos na cidade entre 1961 e 2016, utilizando diferentes métodos da estatística descritiva.

Apesar dos estudos citados e desenvolvidos para o Recife e outras localidades de Pernambuco, nenhum deles aplicam métodos estatísticos que detecte tendência em séries históricas de variáveis climáticas com base na significância.

Dessa forma, este estudo traz uma análise estatística para detecção de tendências em séries temporais de temperatura e precipitação no Recife, utilizando testes estatísticos paramétricos (regressão linear simples) e não paramétricos (Mann-Kendall e Curvatura de Sen). É importante destacar que esta análise poderá servir de referência para um planejamento estratégico das medidas adaptativas em drenagem urbana, considerando os cenários climáticos futuros e previstos, aumentando assim, a resiliência da cidade.

\section{MATERIAIS E MÉTODOS}

\subsection{Caracterização da área de estudo}

O Recife é uma cidade litorânea, localizada na Região Nordeste do Brasil, fortemente urbanizada e constantemente acometida por alagamentos em épocas de chuvas intensas. É a maior área urbana do estado de Pernambuco, banhada a leste pelo Oceano Atlântico e limitada pelos municípios de Olinda, Paulista, Camaragibe, Cabo de Santo Agostinho, Jaboatão dos Guararapes e São Lourenço da Mata (Figura 1). Possui uma área de $218,843 \mathrm{~km}^{2}$ e uma população estimada em 2019 de 1.645.727 hab. O clima é predominantemente quente, com temperatura média de $25^{\circ} \mathrm{C}$, precipitação média anual de $2.447 \mathrm{~mm}$ (período das chuvas concentrado entre março e agosto) e umidade relativa média anual de 80\% (IBGE, 2019; PERNAMBUCO, 2011; RECIFE, 2017). 
É a $16^{\mathrm{a}}$ cidade do mundo mais vulnerável às mudanças climáticas devido às suas características físicas e sociais, à alta densidade populacional do seu litoral, ao elevado índice de impermeabilização do solo e à sua baixa altitude (entre 2 e 4 m). Neste sentido, a cidade já começa a apresentar alguns riscos, principalmente os relacionados com o aumento das precipitações intensas e a elevação do nível do mar (COSTA et al., 2010; PBMC, 2016; RECIFE, 2017).

Com base nos riscos eminentes, as ações e medidas que propiciem uma melhor adaptação do sistema de drenagem do Recife, diante das alterações climáticas, ainda são incipientes e baseadas em iniciativas para a mitigação dos impactos. Silva Junior e Silva (2016) relatam que, basicamente, os planos, estudos e legislações até então existentes, discutem a questão das mudanças climáticas ainda de maneira superficial, sem estabelecer diretrizes efetivas para a implementação de ações concretas para a adaptação.

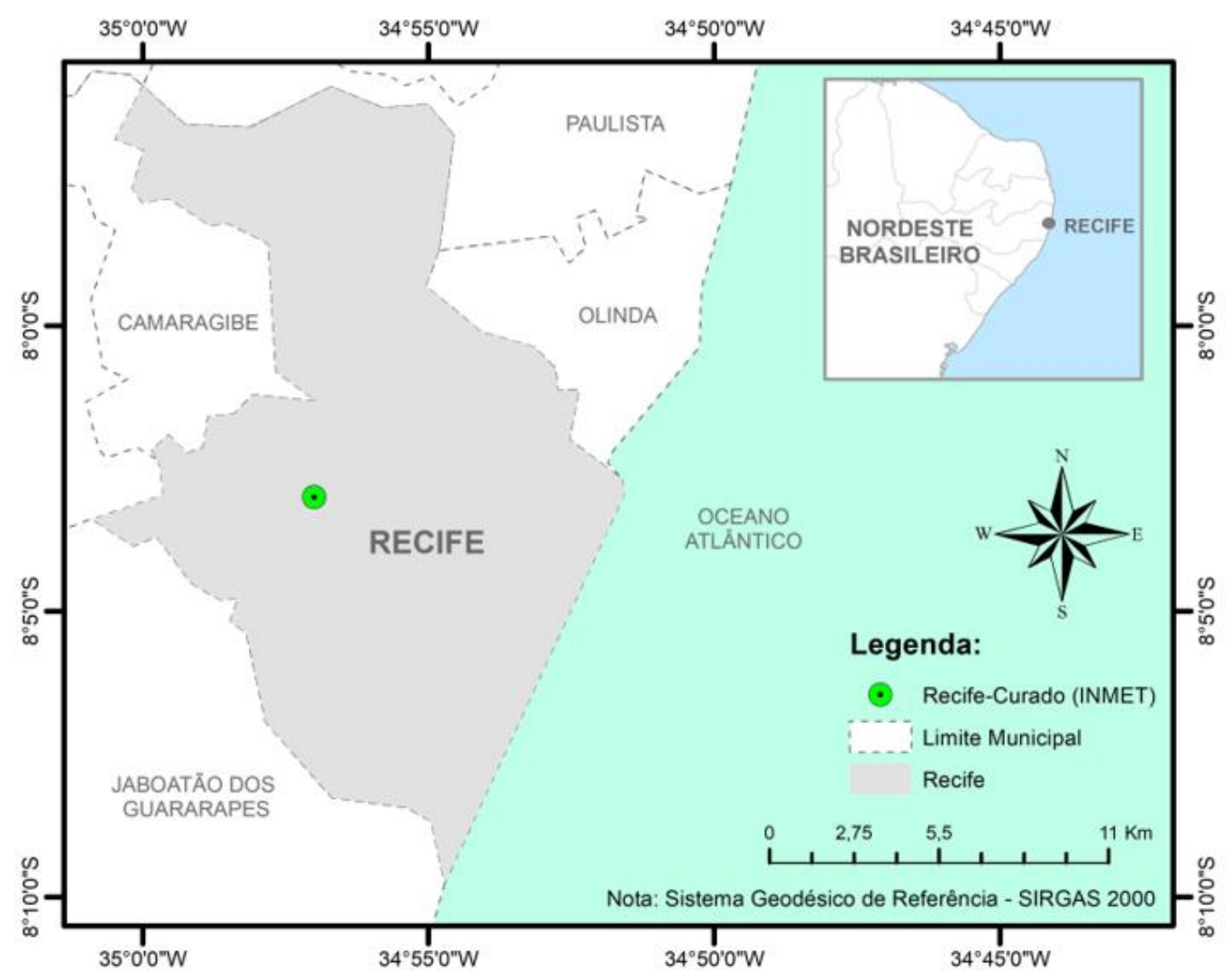

Figura 1 - Localização do município do Recife e da estação do INMET. 


\subsection{Procedimentos adotados}

Neste estudo, foram utilizados os dados diários, mensais e anuais de temperatura (máxima, média e mínima) e de precipitação (máxima e total) da estação climatológica convencional Recife (Curado) - Cód. OMM: 82900, operada pelo INMET, entre 1961 e 2017. Possui coordenadas de $-34,959239^{\circ}$ (longitude) e $-8,05928^{\circ}$ (latitude), e altitude de $11,3 \mathrm{~m}$ (Figura 1). Situa-se em perímetro urbano com baixa taxa de ocupação, no bairro do Curado, próxima à Unidade de Conservação da Natureza (UCN) Mata do Curado.

Os dados foram organizados em planilhas eletrônicas, onde foram sistematizados e analisados. $\mathrm{Na}$ análise exploratória dos dados, foram obtidos os coeficientes de variação (CV) com o intuito de avaliar a variabilidade das séries na escala de tempo definida: $\mathrm{CV} \leq 5 \%$ baixa dispersão, $5 \%<\mathrm{CV}<30 \%$ moderada dispersão e $\mathrm{CV} \geq 30 \%$ alta dispersão. Após isso, a série histórica original foi decomposta em séries parciais com comprimento entre 17 e 20 anos (GUEDES et al., 2005), apenas para avaliar as suas médias em relação ao valor médio da série completa (Tabela 1). De acordo com Pinheiro, Graciano e Severo (2013), o tamanho adotado para as séries parciais permite considerar que os processos cíclicos de curto prazo estejam incorporados, assim como, a existência de estacionaridade na série. Para a série completa, a hipótese de estacionariedade não pode ser garantida, diante da possibilidade de existência de tendências e ciclos na sua evolução temporal.

Tabela 1 - Decomposição das séries históricas de dados.

\begin{tabular}{ccc}
\hline Séries parciais & Período de dados & Anos de dados \\
\hline 1 & $1961-1980$ & 20 anos \\
2 & $1981-2000$ & 20 anos \\
3 & $2001-2017$ & 17 anos
\end{tabular}

Para a identificação de tendência temporal, utilizou-se as séries históricas completas e aplicou-se o método de regressão linear simples e os testes de Mann-Kendall (MK) e de Curvatura de Sen.

No tocante à análise da regressão, os dados de temperatura e precipitação foram distribuídos graficamente e ajustados ao modelo linear do tipo $y=a(x)+b$, onde: " $\mathrm{x}$ " é a variável tempo; "y" é a precipitação ou temperatura; "b" é o valor da reta ajustada para o tempo $\mathrm{t}=0$; e "a" é o coeficiente angular. A tendência da série analisada foi obtida pelo 
comportamento da reta, onde: a $>0$ significa tendência positiva; e a $<0$ indica tendência negativa. Ao realizar esse procedimento, o valor estatístico do $\mathrm{R}^{2}$ (coeficiente de determinação) reflete a qualidade do ajuste efetuado $\left(\mathrm{R}^{2}>0,60 \rightarrow\right.$ Forte; $0,30 \leq \mathrm{R}^{2} \leq 0,60 \rightarrow$ Moderado; $\mathrm{R}^{2}<$ $0,30 \rightarrow$ Fraco; e $\mathrm{R}^{2}=0 \rightarrow$ Nulo).

Os testes não-paramétricos e sequenciais de Mann-Kendall e Curvatura de Sen se apresentam como métodos apropriados para a análise de tendências e/ou mudanças em séries temporais de dados (PINHEIRO; GRACIANO; SEVERO, 2013; SANTOS; NISHIYAMA, 2016). Ambas aplicações neste trabalho foram conduzidas pelo software estatístico XLSTAT 2018, em versão teste, cuja interface é integrada ao Microsoft Excel.

Os níveis de significância dos testes aplicados foram de 5\% e 10\%, caracterizando as tendências em "significante" e "levemente significante", respectivamente (PENEREIRO; FERREIRA, 2011). A hipótese nula - H0 (que indica a não existência de tendência na série de dados) será aceita quando o p-value (nível descritivo do teste) da hipótese alternativa - Ha (que revela tendência na série de dados) não estiver dentro do intervalo de confiança estabelecido pelos níveis de significância.

\section{RESULTADOS E DISCUSSÕES}

\subsection{Análise exploratória dos dados}

As séries temporais de temperaturas mínima, média e máxima (mensal e anual) apresentaram dados com baixa dispersão e pouca variabilidade $(\mathrm{CV} \leq 5 \%)$. Os valores médios de temperatura mínima, média e máxima (mensal e anual) da série parcial 3 (2001-2017) encontram-se acima da média histórica (1961-2017), revelando aumento da variável ao longo do tempo (Tabela 2). Outro indicativo é apresentado nos valores médios anuais das temperaturas analisadas, com médias ascendentes ao longo das três séries parciais consideradas (Tabela 2).

Já as séries temporais de precipitação total e máxima (mensal) apresentaram dados com alta dispersão e grande variabilidade $(\mathrm{CV} \geq 30 \%)$. Observa-se que os valores médios das séries parciais 2 e 3 (1981-2000 e 2001-2017, respectivamente) para os totais precipitados anuais e apenas para o período chuvoso, encontram-se abaixo da média histórica (Tabela 3). Em contrapartida, os valores médios da série parcial 3 (2001-2017), para as precipitações máximas 
anuais e durante o período chuvoso, são maiores que o valor médio da série completa, indicando o aumento dos índices pluviométricos diários (Tabela 3).

Numa análise decadal dos totais precipitados registrados no Recife entre 1961 e 2017, verificou-se que o período de 1964 a 1973 foi o mais chuvoso, com valor médio de 2513,24 mm, enquanto que entre 1990 e 1999 foi menos chuvoso, com 2011,93 mm. Ainda foi possível observar que grande parte dos valores médios nas séries decadais de totais precipitados ficaram abaixo da média histórica $(2290,55 \mathrm{~mm})$ a partir dos anos 90 .

Numa análise gráfica de dados em dispersão (Figura 2), confrontando as variáveis "precipitação" e "temperatura", e contemplando os períodos chuvoso (março a julho) e completo (janeiro a dezembro), fica notório o comportamento das citadas variáveis para os intervalos anuais das séries parciais trabalhadas: 1961-1980; 1981-2000; e 2001-2017.

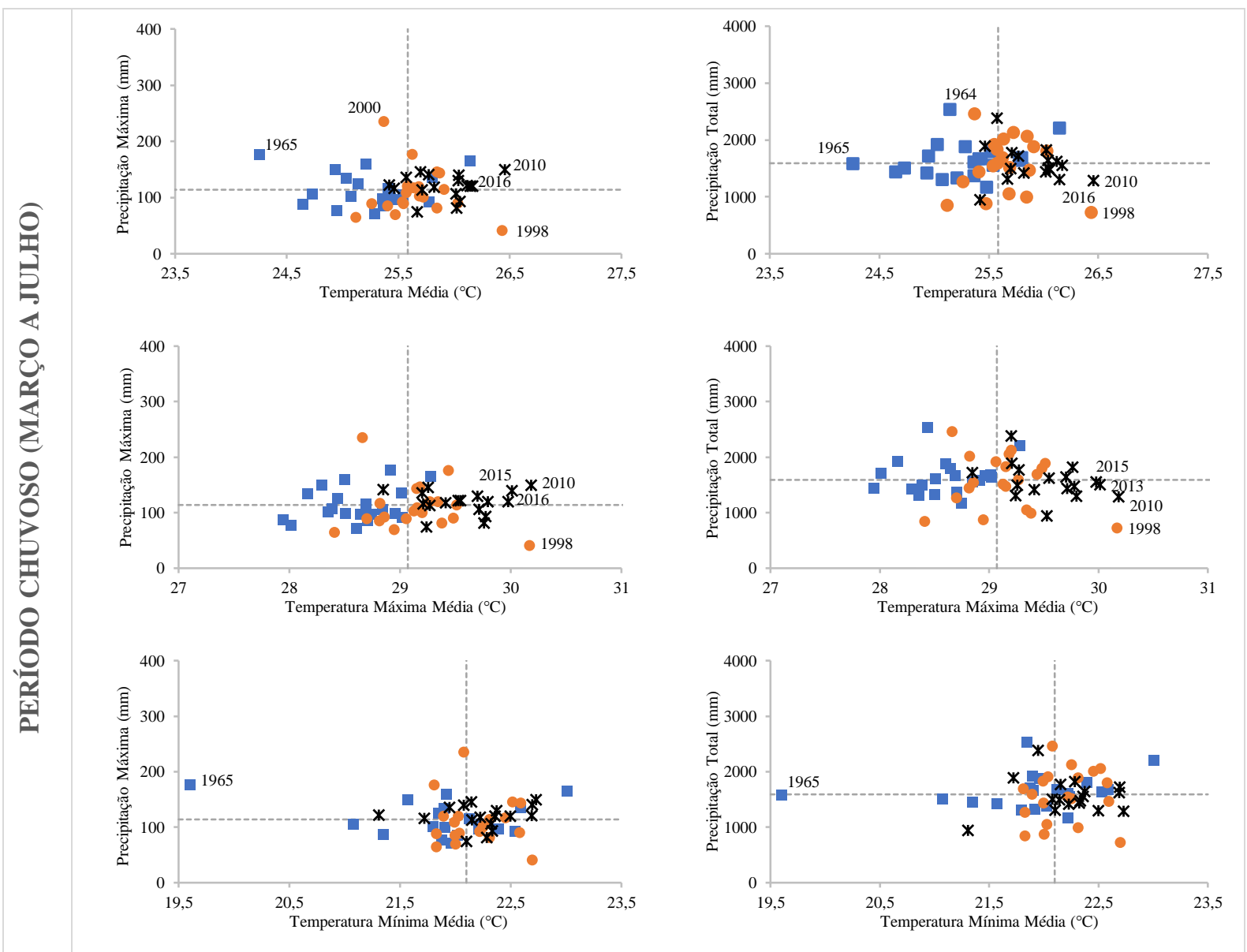



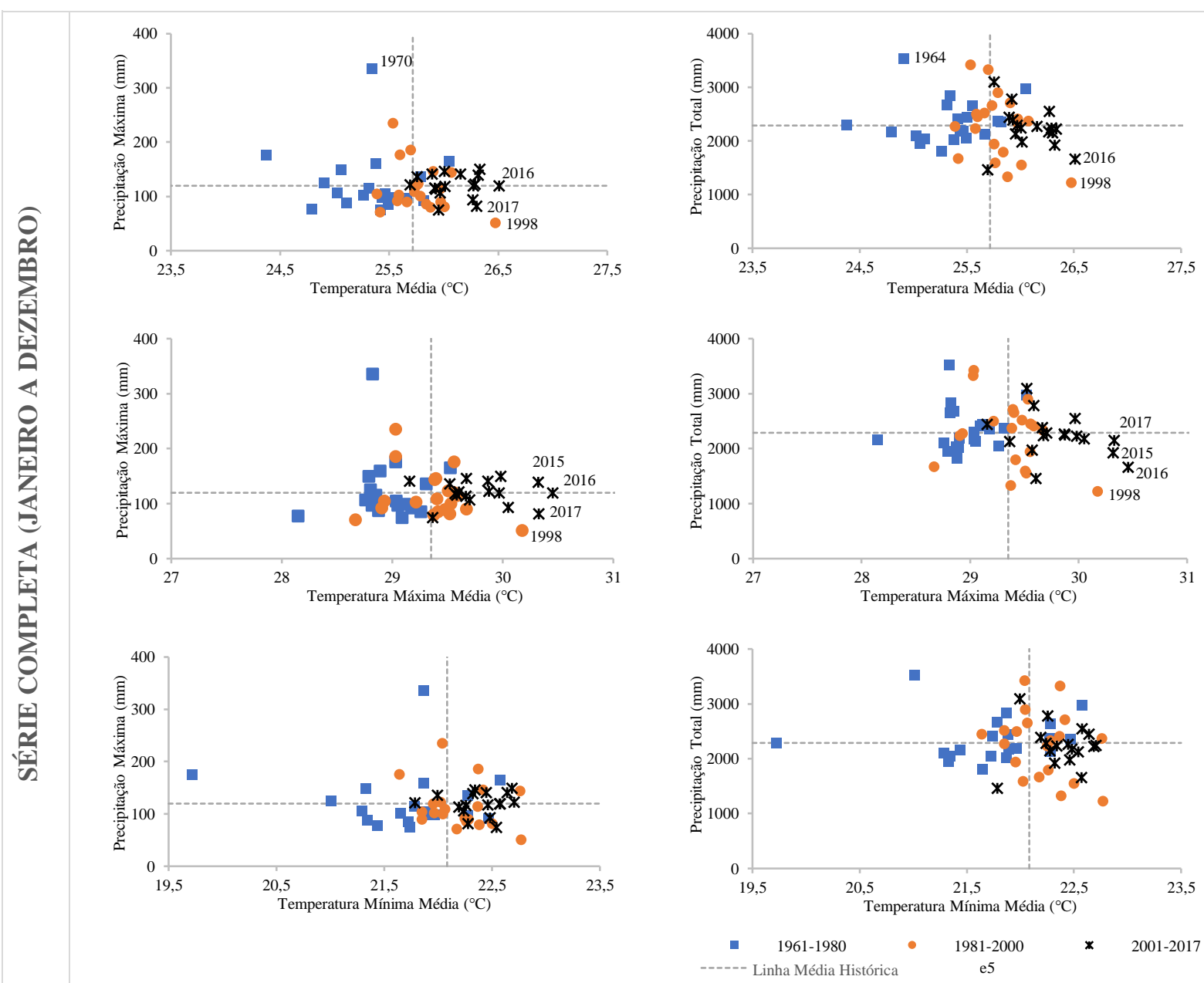

Figura 2 - Gráficos de dispersão com dados anuais de temperatura e precipitação no Recife (1961-2017).

Quando analisado o período chuvoso (março a julho) na Figura 2, evidenciou-se o aumento dos registros médios de temperatura média e máxima nos últimos anos, com valores de temperatura do ar acima da média histórica (1961-2017) em boa parte da série parcial 3 (2001-2017). Já os registros médios de temperatura mínima têm ficado mais concentrados entre 21 e $23{ }^{\circ} \mathrm{C}$, com pouca variabilidade e baixa dispersão em relação à média histórica. Para as precipitações máximas e totais registradas, não é possível evidenciar tendência de aumento ou redução nos seus índices. Entretanto, observou-se que nos últimos anos (2001-2017), os registros de precipitação máxima se mantiveram mais próximos da média histórica, apresentando moderada dispersão $(\mathrm{CV}=19 \%)$.

Ainda sobre a Figura 2 e analisando a série completa (janeiro a dezembro), observou-se que o aumento nos valores médios de temperaturas média, máxima e mínima, nos últimos anos, ficam ainda mais evidentes, com valores de temperatura do ar acima da média histórica (1961 2017) em quase todos os anos da série parcial 3 (2001-2017). Quanto às precipitações máximas 
e totais, o comportamento de seus índices é similar à análise do período chuvoso, sem evidente tendência e com grande parte dos registros de precipitação máxima bem próximos à média histórica na série parcial 3 (2001-2017), com moderada dispersão (CV = 18\%). Nesse caso específico, observou-se redução na dispersão e variabilidade dos registros de precipitação máxima ao longo das três séries parciais (1961-1980 $\rightarrow \mathrm{CV}=48,4 \%$; 1981-2000 $\rightarrow \mathrm{CV}=37 \%$; $2001-2017 \rightarrow \mathrm{CV}=18 \%$ ).

\subsection{Análise de tendências nas séries temporais}

\section{a) Temperatura:}

Em parte das séries temporais de temperaturas mínima, média e máxima (mensal e anual), a regressão linear simples apontou tendência positiva nos dados, com coeficientes angulares maiores que zero $(a>0)$ e coeficientes de determinação variando de moderado a forte. Em contrapartida, a maioria dos modelos lineares não apresentaram tendências significativas estatisticamente, com baixos coeficientes de determinação $\left(R^{2}<0,30\right)$, principalmente nas séries temporais de temperatura mínima (Tabela 2).

Com a aplicação dos testes de Mann-Kendall e de Curvatura de Sen, adotando 5\% de nível de significância, observou-se que os dados analisados apresentaram tendências positivas significantes, com exceção dos meses de junho e julho da série temporal de temperatura mínima ( $p$-value $=0,248$ e 0,069, respectivamente), em que foram aceitas a hipótese nula, $p$-value >

0,05 (Tabela 2). Para 10\% de nível de significância, o mês de julho revela tendência positiva levemente significante, rejeitando a hipótese nula anterior. 
Tabela 2 - Análise estatística nas séries temporais de temperaturas mínima, média e máxima (1961-2017).

\begin{tabular}{|c|c|c|c|c|c|c|c|c|c|c|c|c|c|c|}
\hline \multicolumn{2}{|c|}{ PARAM. ESTATÍSTICOS } & JAN & FEV & MAR & ABR & MAI & JUN & JUL & AGO & SET & OUT & NOV & DEZ & ANUAL \\
\hline \multirow{14}{*}{ 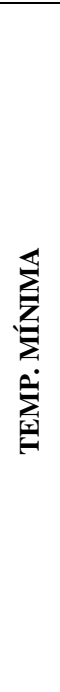 } & \multicolumn{14}{|c|}{1 - Análise Exploratória dos Dados } \\
\hline & $\mathrm{CV}(\%)$ & 4 & 3 & 3 & 3 & 2 & 2 & 3 & 3 & 3 & 4 & 4 & 4 & 2 \\
\hline & Média (1961-2017) & 22,72 & 22,96 & 22,94 & 22,70 & 22,20 & 21,62 & 21,03 & 20,70 & 21,30 & 21,96 & 22,31 & 22,62 & 22,10 \\
\hline & Média (1961-1980) & $22,25 \downarrow$ & $22,52 \downarrow$ & $22,60 \downarrow$ & $22,47 \downarrow$ & $22,03 \downarrow$ & $21,55 \downarrow$ & $20,87 \downarrow$ & $20,34 \downarrow$ & $20,98 \downarrow$ & $21,43 \downarrow$ & $21,62 \downarrow$ & $22,07 \downarrow$ & $21,74 \downarrow$ \\
\hline & Média (1981-2000) & $22,88 \uparrow$ & $23,10 \uparrow$ & $23,06 \uparrow$ & $22,86 \uparrow$ & $22,25 \uparrow$ & $21,63 \uparrow$ & $21,11 \uparrow$ & $20,93 \uparrow$ & $21,35 \uparrow$ & $22,07 \uparrow$ & $22,47 \uparrow$ & $22,65 \uparrow$ & $22,20 \uparrow$ \\
\hline & Média (2001-2017) & $23,06 \uparrow$ & $23,27 \uparrow$ & $23,21 \uparrow$ & $22,78 \uparrow$ & $22,34 \uparrow$ & $21,69 \uparrow$ & $21,13 \uparrow$ & $20,84 \uparrow$ & $21,64 \uparrow$ & $22,46 \uparrow$ & $22,92 \uparrow$ & $23,21 \uparrow$ & $22,38 \uparrow$ \\
\hline & \multicolumn{14}{|c|}{2 - Teste Paramétrico: Regressão Linear Simples } \\
\hline & a (coef. angular) & 0,025 & 0,022 & 0,018 & 0,011 & 0,010 & 0,005 & 0,009 & 0,014 & 0,020 & 0,029 & 0,035 & 0,029 & 0,019 \\
\hline & $\mathrm{R}^{2}$ & 0,261 & 0,211 & 0,217 & 0,095 & 0,087 & 0,023 & 0,064 & 0,114 & 0,249 & 0,385 & 0,456 & 0,286 & 0,363 \\
\hline & \multicolumn{14}{|c|}{3 - Testes Não-Paramétricos: Mann-Kendall e Curvatura de Sem } \\
\hline & Kendall's tau & 0,318 & 0,295 & 0,322 & 0,222 & 0,203 & 0,062 & 0,135 & 0,222 & 0,328 & 0,418 & 0,499 & 0,384 & 0,438 \\
\hline & $p$-value & 0,000 & 0,001 & 0,000 & 0,007 & 0,013 & 0,248 & 0,069 & 0,007 & 0,000 & $<0,0001$ & $<0,0001$ & $<0,0001$ & $<0,0001$ \\
\hline & Sen's slope & 0,020 & 0,016 & 0,015 & 0,010 & 0,007 & 0,003 & 0,006 & 0,012 & 0,015 & 0,024 & 0,031 & 0,025 & 0,016 \\
\hline & Tendência* & $(+)$ & $(+)$ & $(+)$ & $(+)$ & $(+)$ & (null) & (null) & $(+)$ & $(+)$ & $(+)$ & $(+)$ & $(+)$ & $(+)$ \\
\hline \multirow{14}{*}{ 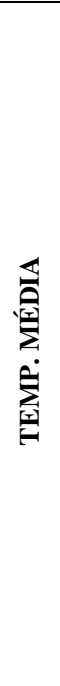 } & \multicolumn{14}{|c|}{1 - Análise Exploratória dos Dados } \\
\hline & $\mathrm{CV}(\%)$ & 2 & 2 & 2 & 2 & 2 & 2 & 2 & 2 & 2 & 2 & 2 & 2 & 2 \\
\hline & Média (1961-2017) & 26,60 & 26,78 & 26,70 & 26,34 & 25,69 & 24,90 & 24,30 & 24,20 & 24,81 & 25,64 & 26,21 & 26,53 & 25,74 \\
\hline & Média (1961-1980) & $26,23 \downarrow$ & $26,36 \downarrow$ & $26,19 \downarrow$ & $25,99 \downarrow$ & $25,35 \downarrow$ & $24,66 \downarrow$ & $24,07 \downarrow$ & $23,89 \downarrow$ & $24,47 \downarrow$ & $25,23 \downarrow$ & $25,72 \downarrow$ & $26,07 \downarrow$ & $25,37 \downarrow$ \\
\hline & Média (1981-2000) & $26,71 \uparrow$ & $26,80 \uparrow$ & $26,83 \uparrow$ & $26,41 \uparrow$ & $25,76 \uparrow$ & $24,95 \uparrow$ & $24,33 \uparrow$ & $24,32 \uparrow$ & $24,86 \uparrow$ & $25,70 \uparrow$ & 26 & $26,50 \downarrow$ & $78 \uparrow$ \\
\hline & Média (2001-2017) & $26,89 \uparrow$ & $27,18 \uparrow$ & $27,13 \uparrow$ & $26,67 \uparrow$ & $26,02 \uparrow$ & $25,10 \uparrow$ & $24,53 \uparrow$ & $24,43 \uparrow$ & $25,16 \uparrow$ & $26,07 \uparrow$ & $26,79 \uparrow$ & $27,10 \uparrow$ & $26,09 \uparrow$ \\
\hline & \multicolumn{14}{|c|}{2 - Teste Paramétrico: Regressão Linear Simples } \\
\hline & a (coef. angular) & 0,020 & 0,022 & 0,026 & 0,020 & 0,018 & 0,012 & 0,013 & 0,015 & 0,020 & 0,023 & 0,028 & 0,026 & 0,020 \\
\hline & $\mathrm{R}^{2}$ & 0,335 & 0,340 & 0,472 & 0,408 & 0,460 & 0,260 & 0,305 & 0,286 & 0,469 & 0,500 & 0,591 & 0,502 & 0,621 \\
\hline & \multicolumn{14}{|c|}{3 - Testes Não-Paramétricos: Mann-Kendall e Curvatura de Sen } \\
\hline & Kendall's tau & 0,384 & 0,413 & 0,509 & 0,439 & 0,490 & 0,344 & 0,377 & 0,391 & 0,514 & 0,520 & 0,596 & 0,519 & 0,619 \\
\hline & p-value & $<0,0001$ & $<0,0001$ & $<0,0001$ & $<0,0001$ & $<0,0001$ & $<0,0001$ & $<0,0001$ & $<0,0001$ & $<0,0001$ & $<0,0001$ & $<0,0001$ & $<0,0001$ & $<0,0001$ \\
\hline & Sen's slope & 0,018 & 0,021 & 0,025 & 0,021 & 0,018 & 0,011 & 0,012 & 0,015 & 0,018 & 0,020 & 0,027 & 0,026 & 0,020 \\
\hline & Tendência* & $(+)$ & $(+)$ & $(+)$ & $(+)$ & $(+)$ & $(+)$ & (i) & $(+)$ & $(+)$ & $(+)$ & $(+)$ & $(+)$ & $(+)$ \\
\hline \multirow{14}{*}{ 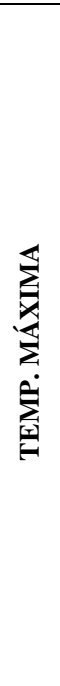 } & \multicolumn{14}{|c|}{1 - Análise Exploratória dos Dados } \\
\hline & $\mathrm{CV}(\%)$ & 2 & 2 & 3 & 2 & 2 & 2 & 2 & 2 & 2 & 2 & 2 & 2 & 1 \\
\hline & Média (1961-2017) & 30,48 & 30,59 & 30,45 & 29,98 & 29,19 & 28,17 & 27,57 & 27,70 & 28,32 & 29,33 & 30,11 & 30,44 & 29,38 \\
\hline & Média (1961-1980) & $30,21 \downarrow$ & $30,21 \downarrow$ & $29,78 \downarrow$ & $29,52 \downarrow$ & $28,67 \downarrow$ & $27,77 \downarrow$ & $27,26 \downarrow$ & $27,43 \downarrow$ & $27,96 \downarrow$ & $29,02 \downarrow$ & $29,83 \downarrow$ & $30,07 \downarrow$ & $29,00 \downarrow$ \\
\hline & Média (1981-2000) & $30,53 \uparrow$ & $30,50 \downarrow$ & $30,6 \uparrow$ & $29,95 \downarrow$ & $29,27 \uparrow$ & $28,28 \uparrow$ & $27,56 \downarrow$ & $27,71 \uparrow$ & $28,38 \uparrow$ & $29,33 \downarrow$ & $29,91 \downarrow$ & $30,35 \downarrow$ & $29,36 \downarrow$ \\
\hline & Média (2001-2017) & $30,73 \uparrow$ & $31,10 \uparrow$ & $31,05 \uparrow$ & $30,56 \uparrow$ & $29,70 \uparrow$ & $28,52 \uparrow$ & $27,94 \uparrow$ & $28,02 \uparrow$ & $28,68 \uparrow$ & $29,68 \uparrow$ & $30,66 \uparrow$ & $30,99 \uparrow$ & $29,80 \uparrow$ \\
\hline & \multicolumn{14}{|c|}{2 - Teste Paramétrico: Regressão Linear Simples } \\
\hline & a (coef. angular) & 0,014 & 0,023 & 0,033 & 0,029 & 0,026 & 0,020 & 0,018 & 0,016 & 0,019 & 0,017 & 0,021 & 0,023 & 0,021 \\
\hline & $\mathrm{R}^{2}$ & 0,160 & 0,238 & 0,409 & 0,467 & 0,554 & 0,494 & 0,359 & 0,309 & 0,384 & 0,264 & 0,317 & 0,372 & 0,609 \\
\hline & \multicolumn{14}{|c|}{3 - Testes Não-Paramétricos: Mann-Kendall e Curvatura de Sen } \\
\hline & Kendall's tau & 0,274 & 0,308 & 0,447 & 0,534 & 0,550 & 0,515 & 0,438 & 0,396 & 0,426 & 0,324 & 0,412 & 0,448 & 0,588 \\
\hline & p-value & 0,001 & 0,000 & $<0,0001$ & $<0,0001$ & $<0,0001$ & $<0,0001$ & $<0,0001$ & $<0,0001$ & $<0,0001$ & 0,000 & $<0,0001$ & $<0,0001$ & $<0,0001$ \\
\hline & Sen's slope & 0,015 & 0,022 & 0,033 & 0,030 & 0,027 & 0,021 & 0,020 & 0,016 & 0,018 & 0,015 & 0,020 & 0,022 & 0,021 \\
\hline & Tendência* & $(+)$ & $(+)$ & $(+)$ & $(+)$ & $(+)$ & $(+)$ & $(+)$ & $(+)$ & $(+)$ & $(+)$ & $(+)$ & $(+)$ & $(+)$ \\
\hline
\end{tabular}

Nota: $\uparrow$ Acima da média histórica (1961-2017); $\downarrow$ Abaixo da média histórica (1961-2017); *Tendência com 5\% de nível de significância; (+) Tendência positiva; (-) Tendência negativa; (null) Hipótese nula, sem tendência. 
Ao longo do período analisado (1961-2017), estima-se que as temperaturas mínima, média e máxima cresceram, aproximadamente, $0,91{ }^{\circ} \mathrm{C}, 1,14{ }^{\circ} \mathrm{C}$ e $1,20{ }^{\circ} \mathrm{C}$, respectivamente. Isso quer dizer que há um aumento anual de $0,016^{\circ} \mathrm{C}$ nas temperaturas mínimas, $0,020{ }^{\circ} \mathrm{C}$ nas temperaturas médias e $0,021{ }^{\circ} \mathrm{C}$ nas temperaturas máximas, corroborando com Souza e Azevedo $(2009 ; 2012)$ que apontou aumentos anuais de $\quad 0,020{ }^{\circ} \mathrm{C}$ nas temperaturas mínimas e $0,022{ }^{\circ} \mathrm{C}$ nas temperaturas máximas, entre os anos de 1961 e 2008 . Os cenários projetados pelo modelo BESM para o período de 2010 a 2100 nas proximidades da Estação Recife (Curado) apontam tendência similar aos obtidos nesta análise, com aumento médio anual de $0,012{ }^{\circ} \mathrm{C}$ para o cenário RCP 4,5 (intermediário) e $0,029{ }^{\circ} \mathrm{C}$ para o RCP 8,5 (pessimista) nas temperaturas médias (NOBRE et al., 2013).

Segundo PMBC (2016), as projeções de temperatura do ar no Recife para os períodos de 2011-2040, 2041-2070 e 2071-2100, geradas pelos registros de 1961 a 1990 a partir de modelos climáticos globais, indicaram aumento na frequência de temperaturas extremas durante o dia e à noite. Para Recife (2019), a partir dos modelos regionalizados (Eta-hadGEM2ES e Eta-MIROC5), no período de 2011-2040 haverá aumento nas temperaturas médias, máximas e mínimas do Recife, com variações entre 3 e 5\% para os cenários intermediário e pessimista (RCP 4,5 e RCP 8,5, respectivamente).

\section{b) Precipitação:}

Nas séries de precipitações máximas e totais (mensal e anual), a regressão linear simples não apontou tendência estatisticamente significante nos dados históricos, diante dos baixos coeficientes de determinação $\left(\mathrm{R}^{2}<0,30\right)$ e dos altos coeficientes de variação (Tabela 3$)$.

Em contrapartida, os testes de Mann-Kendall e Curvatura de Sen mostraram que, apenas para os meses de março e setembro da série temporal de totais precipitados, foi detectada tendência negativa e estatisticamente significativa, rejeitando a hipótese nula. Para as outras unidades de tempo da sobredita série, bem como para as séries temporais de precipitação máxima, foram aceitas a hipótese nula ( $p$-value > 0,05), sem detecção de tendência estatisticamente significante (Tabela 3).

Para os meses de março e setembro, foram estimadas reduções de 106,08 mm (-1,86 $\mathrm{mm} / \mathrm{mês}$ por ano) e 47,48 $\mathrm{mm}$ (-0,84 $\mathrm{mm} / \mathrm{mês}$ por ano), respectivamente, nos totais precipitados ao longo de todo o período analisado (1961-2017). Os cenários projetados pelo modelo BESM (Brazilian Earth System Model) para o período de 2010 a 2100 nas proximidades da Estação 
Recife (Curado) apontam tendência similar aos obtidos nesta análise, com redução média anual de $-1,18 \mathrm{~mm} /$ mês para o cenário RCP 4,5 e -1,81 mm/mês para o RCP 8,5 nas precipitações médias mensais (NOBRE et al., 2013). Corroborando também com os resultados, as projeções dos indicadores de extremos de chuva para os anos de 2011 a 2100, definidas pelo PBMC (2016), indicaram redução nos totais acumulados anuais de chuvas, com índices abaixo da média entre 1961 e 1990.

Tabela 3 - Análise estatística nas séries temporais de precipitação total e máxima (1961-2017).

\begin{tabular}{|c|c|c|c|c|c|c|c|c|c|c|c|c|c|c|c|}
\hline \multicolumn{2}{|c|}{ PARAM. ESTATÍSTICOS } & JAN & FEV & MAR & $\overline{\mathrm{ABR}}$ & MAI & JUN & JUL & $\mathrm{AGO}$ & SET & OUT & $\mathrm{NOV}$ & DEZ & $\mathrm{PCH}^{*}$ & ANUAL \\
\hline \multirow{14}{*}{ 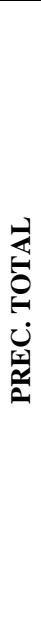 } & \multicolumn{15}{|c|}{1 - Análise Exploratória dos Dados } \\
\hline & $\mathrm{CV}(\%)$ & 68 & 74 & 56 & 57 & 41 & 36 & 39 & 51 & 67 & 76 & 67 & 72 & 23 & 21 \\
\hline & Média (1961-2017) & 103,91 & 134,26 & 234,31 & 292,12 & 319,84 & 387,97 & 356,61 & 199,27 & 109,23 & 57,92 & 38,39 & 58,62 & 1590,84 & 2290,55 \\
\hline & Média (1961-1980) & $98,68 \downarrow$ & $148,0 \uparrow$ & $282,6 \uparrow$ & $303,9 \uparrow$ & $312,1 \downarrow$ & $393,4 \uparrow$ & $358,5 \uparrow$ & $189,3 \downarrow$ & $129,6 \uparrow$ & $59,42 \uparrow$ & $37,4 \downarrow$ & $58,81 \uparrow$ & $1650 \uparrow$ & $2370 \uparrow$ \\
\hline & Média (1981-2000) & $105,8 \uparrow$ & $123,0 \downarrow$ & $210,3 \downarrow$ & $298,8 \uparrow$ & $322,2 \uparrow$ & $345,3 \downarrow$ & $378,8 \uparrow$ & $219,5 \uparrow$ & $109,2 \downarrow$ & $60,77 \uparrow$ & $38,6 \uparrow$ & $49,53 \downarrow$ & $1555 \downarrow$ & $2262 \downarrow$ \\
\hline & Média (2001-2017) & $107,6 \uparrow$ & $132,2 \downarrow$ & $205,8 \downarrow$ & $270,4 \downarrow$ & $326,1 \uparrow$ & $431,8 \uparrow$ & $328,3 \downarrow$ & $187,3 \downarrow$ & $85,28 \downarrow$ & $52,82 \downarrow$ & $39,31 \uparrow$ & $69,09 \uparrow$ & $1562 \downarrow$ & $2236 \downarrow$ \\
\hline & \multicolumn{15}{|c|}{2 - Teste Paramétrico: Regressão Linear Simples } \\
\hline & a (coef. angular) & 0,025 & $-0,727$ & $-2,265$ & $-1,016$ & 0,463 & 0,304 & $-0,681$ & $-0,264$ & $-0,873$ & $-0,029$ & 0,113 & 0,244 & $-3,194$ & $-4,717$ \\
\hline & $\mathrm{R}^{2}$ & 0,000 & 0,014 & 0,080 & 0,010 & 0,003 & 0,001 & 0,006 & 0,002 & 0,038 & 0,000 & 0,005 & 0,009 & 0,020 & 0,026 \\
\hline & \multicolumn{15}{|c|}{3 - Testes Não-Paramétricos: Mann-Kendall e Curvatura de Sem } \\
\hline & Kendall's tau & 0,016 & $-0,105$ & $-0,195$ & $-0,070$ & 0,010 & 0,050 & $-0,033$ & $-0,010$ & $-0,157$ & $-0,021$ & 0,084 & 0,050 & $-0,105$ & $-0,096$ \\
\hline & p-value & 0,433 & 0,126 & 0,016 & 0,222 & 0,456 & 0,293 & 0,358 & 0,456 & 0,043 & 0,410 & 0,178 & 0,293 & 0,124 & 0,148 \\
\hline & Sen's slope & 0,096 & $-0,820$ & $-1,861$ & $-1,084$ & 0,092 & 0,653 & $-0,398$ & 0,099 & $-0,833$ & 0,057 & 0,153 & 0,136 & $-3,081$ & $-4,001$ \\
\hline & Tendência** & (null) & (null) & $(-)$ & (null) & (null) & (null) & (null) & (null) & $(-)$ & (null) & (null) & (null) & (null) & (null) \\
\hline \multirow{14}{*}{ 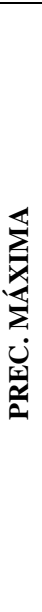 } & \multicolumn{15}{|c|}{ 1-Análise Exploratória dos Dados } \\
\hline & $\mathrm{CV}(\%)$ & 78 & 72 & 55 & 54 & 49 & 43 & 46 & 93 & 76 & 91 & 70 & 100 & 28 & 37 \\
\hline & Média (1961-2017) & 33,78 & 43,06 & 63,24 & 65,37 & 70,84 & 84,75 & 70,58 & 51,21 & 30,24 & 20,57 & 13,86 & 25,48 & 113,92 & 119,75 \\
\hline & Média (1961-1980) & $32,82 \downarrow$ & $44,04 \uparrow$ & $68,40 \uparrow$ & $58,44 \downarrow$ & $66,07 \downarrow$ & $89,56 \uparrow$ & $75,15 \uparrow$ & $58,31 \uparrow$ & $33,34 \uparrow$ & $21,37 \uparrow$ & $14,40 \uparrow$ & $21,64 \downarrow$ & $113,9 \uparrow$ & $124,22 \uparrow$ \\
\hline & Média (1981-2000) & $38,54 \uparrow$ & $39,86 \downarrow$ & $61,03 \downarrow$ & $70,40 \uparrow$ & $73,59 \uparrow$ & $73,26 \downarrow$ & $66,84 \downarrow$ & $55,37 \uparrow$ & $30,67 \uparrow$ & $20,37 \downarrow$ & $12,47 \downarrow$ & $21,04 \downarrow$ & $109,4 \downarrow$ & $115,06 \downarrow$ \\
\hline & Média (2001-2017) & $29,26 \downarrow$ & $45,75 \uparrow$ & $59,77 \downarrow$ & $67,60 \uparrow$ & $73,21 \uparrow$ & $92,61 \uparrow$ & $69,59 \downarrow$ & $37,98 \downarrow$ & $26,10 \downarrow$ & $19,88 \downarrow$ & $14,86 \uparrow$ & $35,22 \uparrow$ & $119,3 \uparrow$ & $120,01 \uparrow$ \\
\hline & \multicolumn{15}{|c|}{2 - Teste Paramétrico: Regressão Linear Simples } \\
\hline & a (coef. angular) & $-0,147$ & 0,047 & $-0,215$ & 0,266 & 0,195 & $-0,010$ & $-0,166$ & $-0,453$ & $-0,174$ & $-0,006$ & 0,053 & 0,307 & 0,069 & $-0,125$ \\
\hline & $\mathrm{R}^{2}$ & 0,008 & 0,001 & 0,010 & 0,016 & 0,009 & 0,000 & 0,007 & 0,025 & 0,016 & 0,000 & 0,008 & 0,039 & 0,001 & 0,002 \\
\hline & \multicolumn{15}{|c|}{3 - Testes Não-Paramétricos: Mann-Kendall e Curvatura de Sen } \\
\hline & Kendall's tau & $-0,014$ & $-0,040$ & $-0,066$ & 0,102 & 0,041 & 0,031 & $-0,078$ & $-0,103$ & $-0,099$ & 0,051 & 0,114 & $-0,001$ & 0,073 & 0,055 \\
\hline & p-value & 0,441 & 0,331 & 0,233 & 0,132 & 0,330 & 0,368 & 0,197 & 0,129 & 0,138 & 0,289 & 0,108 & 0,495 & 0,214 & 0,277 \\
\hline & Sen's slope & $-0,028$ & $-0,093$ & $-0,208$ & 0,299 & 0,099 & 0,079 & $-0,222$ & $-0,201$ & $-0,127$ & 0,049 & 0,067 & 0,000 & 0,216 & 0,200 \\
\hline & Tendência** & (null) & (null) & (null) & (null) & (null) & (null) & (null) & (null) & (null) & (null) & (null) & (null) & (null) & (null) \\
\hline
\end{tabular}

Nota: $\uparrow$ Acima da média histórica (1961-2017); $\downarrow$ Abaixo da média histórica (1961-2017); *Período chuvoso (março, abril, maio, junho e julho); **Tendência com 5\% de nível de significância; (+) Tendência positiva; (-) Tendência negativa; (null) Hipótese nula, sem tendência. 


\subsection{Análise de tendências na ocorrência de precipitações extremas}

Boa parte da literatura, que tem como enfoque as precipitações extremas no meio urbano, consideram que chuvas a partir de $30 \mathrm{~mm}$ em $24 \mathrm{~h}$ já podem causar impactos na rotina de uma cidade (FONSECA, 2017; NUNES; PINTO; BAPTISTA, 2018; PBMC, 2016; SOUZA; AZEVEDO; ARAÚJO, 2012; WANDERLEY et al., 2018). Os valores extremos de chuva em 24 h nas estações do INMET, como a Recife (Curado), registram os maiores acumulados de chuva entre $9 \mathrm{~h}$ do dia anterior e $9 \mathrm{~h}$ do dia em questão.

Ao analisar a frequência desses eventos em Recife, foram encontrados um total de 1140 registros entre 1961 e 2017, caracterizando uma média de 20 ocorrências por ano. Apesar desse número, tem-se observado uma redução na ocorrência de tais eventos ao longo do tempo. Estatisticamente, o teste de Mann-Kendall comprova essa tendência negativa e levemente significante (Tabela 4).

Tabela 4 - Frequência e tendência da ocorrência de dias com precipitações acima de $30 \mathrm{~mm}$.

\begin{tabular}{|c|c|c|c|c|c|c|c|c|c|}
\hline \multirow{2}{*}{$\begin{array}{r}\text { Faixa de } \\
\text { Precipitação }\end{array}$} & \multicolumn{4}{|c|}{ Total de dias } & \multicolumn{2}{|c|}{$\begin{array}{l}\text { Regressão } \\
\text { Linear }\end{array}$} & \multicolumn{3}{|c|}{ Teste de Mann-Kendall } \\
\hline & $\begin{array}{l}1961- \\
1980\end{array}$ & $\begin{array}{l}1981- \\
2000\end{array}$ & $\begin{array}{l}2001- \\
2017\end{array}$ & $\begin{array}{l}\text { 1961- } \\
2017\end{array}$ & $\mathbf{a}$ & $\mathbf{R}^{2}$ & $\begin{array}{r}p- \\
\text { value }\end{array}$ & $5 \% *$ & $10 \% * *$ \\
\hline $30-39,9 \mathrm{~mm}$ & 149 & 134 & 120 & 403 & $\begin{array}{c}- \\
0,016\end{array}$ & $\begin{array}{l}0,0 \\
11\end{array}$ & $\begin{array}{l}0,1 \\
47\end{array}$ & (null) & (null) \\
\hline $40-49,9 \mathrm{~mm}$ & 93 & 82 & 63 & 238 & $\begin{array}{c}- \\
0,020\end{array}$ & $\begin{array}{l}0,0 \\
26\end{array}$ & $\begin{array}{l}0,0 \\
68\end{array}$ & (null) & $(-)$ \\
\hline $50-59,9 \mathrm{~mm}$ & 62 & 53 & 38 & 153 & $\begin{array}{r}- \\
0,017\end{array}$ & $\begin{array}{l}0,0 \\
21\end{array}$ & $\begin{array}{l}0,1 \\
26\end{array}$ & (null) & (null) \\
\hline $60-69,9 \mathrm{~mm}$ & 45 & 40 & 31 & 116 & $\begin{array}{c}- \\
0,019\end{array}$ & $\begin{array}{l}0,0 \\
53\end{array}$ & $\begin{array}{l}0,0 \\
35\end{array}$ & $(-)$ & $(-)$ \\
\hline $70-79,9 \mathrm{~mm}$ & 25 & 33 & 22 & 80 & $\begin{array}{c}- \\
0,004\end{array}$ & $\begin{array}{l}0,0 \\
02\end{array}$ & $\begin{array}{l}0,3 \\
81\end{array}$ & (null) & (null) \\
\hline $80-89,9 \mathrm{~mm}$ & 17 & 14 & 14 & 45 & $\begin{array}{c}- \\
0,001\end{array}$ & $\begin{array}{l}0,0 \\
00\end{array}$ & $\begin{array}{l}0,3 \\
00\end{array}$ & (null) & (null) \\
\hline $90-99,9 \mathrm{~mm}$ & 13 & 7 & 10 & 30 & $\begin{array}{r}- \\
0,003\end{array}$ & $\begin{array}{l}0,0 \\
05\end{array}$ & $\begin{array}{l}0,3 \\
02\end{array}$ & (null) & (null) \\
\hline$\geq 100 \mathrm{~mm}$ & 23 & 26 & 26 & 75 & $\begin{array}{l}0,0 \\
11\end{array}$ & $\begin{array}{l}0,0 \\
19\end{array}$ & $\begin{array}{l}0,0 \\
77\end{array}$ & (null) & $(+)$ \\
\hline$\geq 30 \mathrm{~mm}$ & 427 & 389 & 324 & 1140 & $\begin{array}{r}- \\
0,068\end{array}$ & $\begin{array}{l}0,0 \\
36\end{array}$ & $\begin{array}{l}0,0 \\
83\end{array}$ & (null) & $(-)$ \\
\hline
\end{tabular}

Nota: *Tendência significante; **Tendência levemente significante; (+) Tendência positiva; (-) Tendência negativa; (null) Hipótese nula, sem tendência. 
Ao discretizar os registros pluviométricos em faixas de precipitação, observa-se na Tabela 4, a partir da regressão linear simples, que os coeficientes angulares são negativos ( 0 ) em todos os intervalos de altura de chuva, com exceção da faixa $\geq 100 \mathrm{~mm}$. Apesar do modelo linear indicar tendência, estatisticamente, ela não é significante diante dos baixos coeficientes de determinação $\left(\mathrm{R}^{2}\right)$.

Com a aplicação do teste de Mann-Kendall, com níveis de significância de 5\% e 10\%, observou-se que há tendência negativa para as faixas de precipitação 40-49,9 mm e 60-69,9 $\mathrm{mm}$. Para precipitações acima de $100 \mathrm{~mm}$ em $24 \mathrm{~h}$, o teste revelou tendência positiva e levemente significante, corroborando com os resultados de Wanderley et al. (2018), Silva, Montenegro e Souza (2017), PBMC (2016) e Souza e Azevedo (2012) que indicaram haver aumento na ocorrência de chuvas extremamente fortes concentradas em um dia ( $\geq 100 \mathrm{~mm})$. Para as outras faixas de precipitação foram aceitas a hipótese nula, sem identificação de tendência significante ou levemente significante.

Analisando apenas as precipitações extremas com índices acima de 100 mm, observouse que $81 \%$ dos eventos registrados ocorreram durante o período chuvoso, entre os meses de março e julho. Wanderley et al. (2018) também identificaram que os eventos de chuvas fortes a extremamente fortes no Recife ( $\geq 25 \mathrm{~mm}$ ) se concentram, em sua maioria, nos meses de março e julho. Apesar disso, o maior índice pluviométrico foi registrado em agosto de 1970, com 335,8 $\mathrm{mm}$ em $24 \mathrm{~h}$. A média histórica das precipitações extremas ( $\geq 100 \mathrm{~mm}$ ) é 126,67 mm, enquanto que as médias das séries parciais 1, 2 e 3 são 133,92 mm, 129,07 mm e 120,03 mm, respectivamente.

Numa análise dos eventos de chuva acima de $30 \mathrm{~mm}$ em dias consecutivos, verificouse uma redução da ocorrência desses eventos ao longo do período analisado (1961-1980 $\rightarrow 81$ eventos; 1981-2000 $\rightarrow 59$ eventos; 2001-2017 $\rightarrow 42$ eventos). A maior parte desses eventos ocorreram em 2 dias (1961-1980 $\rightarrow 60$ eventos; 1981-2000 $\rightarrow 48$ eventos; 2001-2017 $\rightarrow 37$ eventos), porém há registro de evento com 7 dias consecutivos de chuva acima $30 \mathrm{~mm}$, ocorrido em 20 a 26 de abril de 1970 com total de $317,80 \mathrm{~mm}$.

A Figura 3 mostra os dados em dispersão a partir do confronto entre as variáveis "número de ocorrências de eventos de 2 dias por ano" versus "totais médios precipitados entre tais eventos". Nela é possível observar que, nos últimos anos (2001-2017), o número de eventos com 2 dias consecutivos (considerando chuvas acima de $30 \mathrm{~mm}$ ) têm ficado abaixo da média histórica de ocorrências, que é 2,84. Em contrapartida, grande parte desses eventos 
apresentaram maiores totais precipitados, com valores bem acima da média histórica $(114,83$ mm), sugerindo que, nos últimos anos (2001-2017), houve redução na quantidade de eventos com 2 dias consecutivos no ano, mas com maiores índices de chuvas acumuladas.

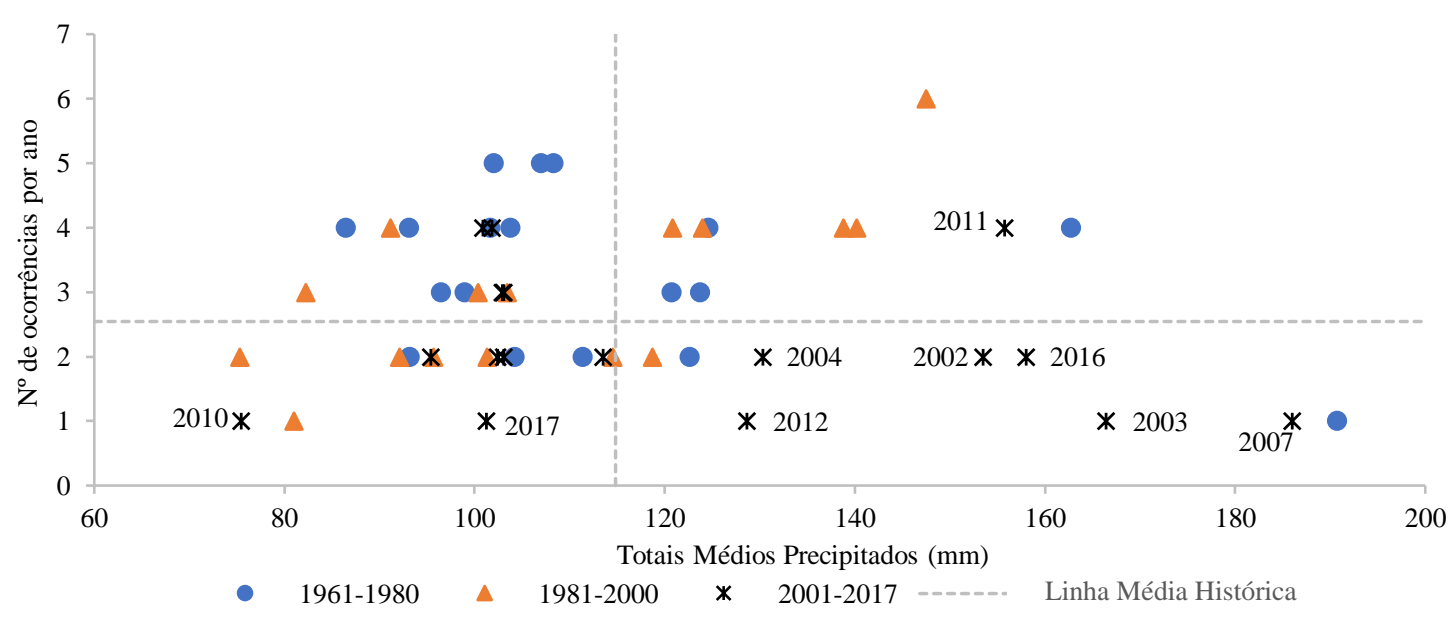

Figura 3 - Gráfico de dispersão para eventos de 2 dias consecutivos com chuvas acima de $30 \mathrm{~mm}$.

\section{CONSIDERAÇÕES FINAIS}

As tendências detectadas nas séries temporais das variáveis climatológicas analisadas, não podem ser atribuídas apenas às mudanças climáticas globais. Isso porque outros vários fatores, em nível local, podem ter contribuído para a modificação do clima no Recife, como por exemplo, a supressão de áreas vegetadas, a expansão e o adensamento urbano. Neste sentido, políticas públicas que disseminem mais espaços vegetados, nãn có snntrihıem para o controle de inundações, proporcionado pela infiltração e pela interceptação vegetal, como também favorecem um microclima urbano mais ameno e saudável.

As séries temporais associadas às temperaturas mínima, média e máxima apontaram tendência positiva e estatisticamente significante, indicando aumento desta variável, em torno de $1{ }^{\circ} \mathrm{C}$, ao longo de 57 anos de dados. A partir dos anos 2000, os valores médios de temperatura mínima, média e máxima (mensal e anual) encontram-se acima da média histórica (1961-2017). Esse aumento agrava ainda mais a formação de ilhas de calor na cidade, resultado do intenso processo de urbanização e verticalização.

Nas séries de precipitações máximas e totais (mensal e anual), a regressão linear simples não apontou tendência significante de redução, uma vez que os modelos lineares apresentaram baixos coeficientes de determinação. Em contrapartida, os testes de Mann-Kendall e Curvatura 
de Sen mostraram que, apenas para os meses de março e setembro, foi detectada tendência negativa e estatisticamente significante nas séries temporais dos totais precipitados.

Quanto à frequência dos eventos extremos, com precipitações acima de $100 \mathrm{~mm}$ em 24 $\mathrm{h}$, foi detectada tendência positiva e levemente significante. Constatou-se, também, uma redução na quantidade de eventos de 2 dias consecutivos no ano (com precipitações acima de $30 \mathrm{~mm}$ ), porém com maiores índices de chuvas acumuladas. Esse resultado acende um sinal de alerta para o Recife, que já possui um sistema de drenagem fragilizado e altamente vulnerável às oscilações de maré. Com esse cenário, a adoção de medidas que contribuam para a adaptação do sistema de drenagem frente às mudanças climáticas se faz necessária e urgente, a fim de tornar a cidade do Recife mais resiliente.

\section{REFERÊNCIAS}

COSTA, M. B. S. F.; MALlMANN, D. L. B.; PONTES, P. M.; ARAÚJO, M. Vulnerability and impacts related to the rising sea level in the Metropolitan Center of Recife, Northeast Brazil. Pan-American Journal of Aquatic Sciences, v. 5, n. 2, p. 341- 349, 2010.

FONSECA, R. G. Risco hidrológico: precipitações extremas, enchentes e alagamentos na cidade de Ituiutaba (MG). Dissertação (Mestrado) - Universidade Estadual Paulista, Instituto de Geociências e Ciências Exatas, 2017, 149 p.

GUEDES, R. L.; ANDREOLI, R. V.; KAYANO, M. T.; OYAMA, M. D.; ALVES, M. A. S. Série temporal de precipitação mensal de Fortaleza, Brasil: comparação entre observações e dados de reanálise do NCEP/NCAR. Revista Brasileira de Meteorologia, v. 20, n. 1, p. 83$92,2005$.

IBGE. Estimativa populacional do Recife em 2019. Disponível em: <https://www.ibge.gov.br/cidades-e-estados/pe/recife.html>. Acesso em: 5 de nov. 2019.

IPCC. AR5 Synthesis Report: Climate Change 2014. Disponível em: 〈https://www.ipcc.ch/report/ar5/syr/>. Acesso em: 08 de mai. 2018. 
MEDEIROS, R. M.; HOLANDA, R. M.; SILVA, V. P. Tendências pluviais e análise da média móvel para São Bento do Una-PE, Brasil. Revista de Geografia (Recife), v. 35, n. 5, p. 38-54, 2018.

MESQUITA, D. A.; FERNANDES, L. L.; ROCHA, G. S.; SILVA, M. N. A.; SILVEIRA, R. N. P. O. Análise de tendência em série temporal, estação pluviométrica São Francisco, Laranjal do Jari - AP. In: Congresso ABES FENASAN 2017, 2017, São Paulo, Anais..., São Paulo: ABES, 2017. p. 1-6.

MigueZ, M. G.; FERnANDES, L. C.; AZEVEDO, J. P. S.; MAGAlhãeS, L. P. C. Vulnerabilidades da infraestrutura de drenagem urbana e os efeitos das mudanças climáticas na Região Metropolitana do Rio de Janeiro. In: NOBRE, C.; YOUNG, A.; GUSMÃO, P. P. (Org.). Megacidades, vulnerabilidades e mudanças climáticas: Região Metropolitana do Rio de Janeiro, Rio de Janeiro: INPE/UNICAMP/UFRJ, 2011. p. 123-144.

NOBRE, P.; SIQUEIRA, L. S. P.; ALMEIDA, R. A. F.; MALAGUTTI, M.; GIAROLLA, E.; CASTElÃO, G. P.; BOtTinO, M. J.; KUBOTA, P.; FigUEROA, S. N.; COSTA, M. C.; BAPTISTA JR, M.; IRBER JR, L.; MARCONDES, G. G. Climate Simulation and Change in the Brazilian Climate Model. Journal of Climate, v. 26, n. 17, p. 6716-6732, 2013.

NUNES, A. A.; PINTO, E. J. A.; BAPTISTA, M. B. Detection of trends for extreme events of precipitation in the Metropolitan Region of Belo Horizonte through statistical methods. Revista Brasileira de Recursos Hídricos, v. 23, n. 9, p. 1-13, 2018.

PBMC. Impacto, vulnerabilidade e adaptação das cidades costeiras brasileiras às mudanças climáticas: Relatório Especial do Painel Brasileiro de Mudanças Climáticas. Rio de Janeiro, 2016. 184 p.

PENEREIRO, J. C.; FERREIRA, D. H. L. Estatística apoiada pela tecnologia: uma proposta para identificar tendências climáticas. Acta Scientiae, v. 13, n. 1, p. 87-105, 2011.

PERNAMBUCO. Secretaria de Meio Ambiente e Sustentabilidade. Plano Estadual de Mudanças Climáticas. Recife, 2011. 94 p. 
PINHEIRO, A.; GRACIANO, R. L. G.; SEVERO, D. L. Tendência das séries temporais de precipitação da região sul do Brasil. Revista Brasileira de Meteorologia, v. 28, n. 3, p. 281$290,2013$.

RECIFE. Secretaria de Meio Ambiente e Sustentabilidade. Índice de Risco à Mudança do Clima em Recife - PE. Recife, 2019. 165 p.

RECIFE. Secretaria de Meio Ambiente e Sustentabilidade. Projeto Pegada de Cidades: Inventário de Emissão de Gases de Efeito Estufa (2012-2015) e Pegada Hídrica (2015) do Recife. Recife, 2017.71 p.

ROCHA, G. S.; FERNANDES, L. L.; SILVEIRA, R. N. P. O.; SILVA, M. N. A.; MESQUITA, D. A. Análise de tendência da precipitação pluviométrica através de métodos estatísticos não paramétricos para o município de Monte do Carmo/TO. In: Congresso ABES FENASAN 2017, 2017, São Paulo, Anais... São Paulo: ABES, 2017. p. 1-7.

SALVIANO, M. F.; GROPPO, J. D.; PELlEGRINO, G. Q. Análise de tendências em dados de precipitação e temperatura no Brasil. Revista Brasileira de Meteorologia, v. 31, n. 1, p. 6473, 2016.

SANTOS, V. O.; NISHIYAMA, L. Tendências hidrológicas no alto curso da bacia hidrográfica do rio Uberaba, em Minas Gerais. Caminhos de Geografia, v. 17, n. 58, p. 196-212, 2016.

SILVA, R. O. B.; MONTENEGRO, S. M. G. L.; SOUZA, W. M. Tendências de mudanças climáticas na precipitação pluviométrica nas bacias hidrográficas do estado de Pernambuco. Engenharia Sanitária e Ambiental, v. 22, n. 3, p. 579-589, 2017.

SILVA JUNIOR, M. A. B.; SILVA, S. R. Impactos da urbanização e das alterações climáticas no sistema de drenagem do Recife/PE. Revista Brasileira de Geografia Física, v. 9, n. 2, p. 353-367, 2016. 
SOUZA, W. M.; AZEVEDO, P. V. Avaliação de tendências das temperaturas em Recife-PE: mudanças climáticas ou variabilidade? Engenharia Ambiental - Espírito Santo do Pinhal, v. 6, n. 3, p. 462-472, 2009.

SOUZA, W. M.; AZEVEDO, P. V. Índices de Detecção de Mudanças Climáticas Derivados da Precipitação Pluviométrica e das Temperaturas em Recife-PE. Revista Brasileira de Geografia Física, v. 5, n. 1, p. 143-159, 2012.

SOUZA, W. M.; AZEVEDO, P. V.; ARAÚJO, L. E. Classificação da Precipitação Diária e Impactos Decorrentes dos Desastres Associados às Chuvas na Cidade do Recife-PE. Revista Brasileira de Geografia Física, v. 5, n. 2, p. 250-268, 2012.

WANDERLEY, H. S.; JUSTINO, F. B.; SEDIYAMA, G. C. Tendência da Temperatura e Precipitação na Península Antártica. Revista Brasileira de Meteorologia, v. 31, n. 2, p. 114$121,2016$.

WANDERLEY, L. S. A.; NÓBREGA, R. S.; MOREIRA, A. B.; ANJOS, R. S.; ALMEIDA, C. A. P. As chuvas na cidade do Recife: uma climatologia de extremos. Revista Brasileira de Climatologia, vol. 22, p. 149-164, 2018. 